\title{
Interpretation of NCCN guidelines for hepatobiliary tumors V3 update in 2021
}

\author{
Lingxiao Xu, Jun Zhou \\ Department of Gastrointestinal Oncology, Peking University Cancer Hospital \& Institute, Beijing, China \\ Correspondence to: Jun Zhou. Department of Gastrointestinal Oncology, Peking University Cancer Hospital \& Institute, Beijing, China. \\ Email: joelbmu@126.com.
}

Submitted Aug 31, 2021. Accepted for publication Sep 12, 2021.

doi: $10.21037 / \mathrm{hbsn}-21-355$

View this article at: https://dx.doi.org/10.21037/hbsn-21-355

Interpretation of NCCN guidelines for hepatobiliary tumors V3 update in 2021 (1).

Hepatobiliary tumors are a general term for a large group of tumors, including liver cancer and biliary tract tumors. It is rare, highly aggressive and has a poor prognosis.

Biliary tract tumors are highly heterogeneous, and the chemotherapy effect of unresectable tumors is poor. The objective response rate (ORR) of first-line chemotherapy is $20-30 \%$, while ORR of second-line chemotherapy is only about $5 \%$. As one of the most authoritative guidelines, NCCN proposed some new medical treatment recommendations, and these updates mainly focused on targeted therapy and immunotherapy, with relatively few updates on chemotherapy this year.

In biliary tract cancer, the update embodies the concept of precision treatment of biliary tract tumors. In this update, it is pointed out that infigratinib can be used for FGFR2 fused or rearranged cholangiocarcinoma as the second-line therapy and above after disease progression. The updated content is based on the phase 2 clinical trial of infigratinib (BGJ398) (2). As of March 31, 2020, a total of 108 adult patients with advanced/metastatic cholangiocarcinoma who had experienced disease progression following at least one previous treatment were included in the study. They were given infigratinib $125 \mathrm{mg}$ po (28-day cycle; 3 weeks on, 1 week off). The 108 participants includes 83 patients with FGFR2 gene fusion/rearrangement who had not received FGFR inhibitor treatment before, with an ORR of $23.1 \%$ (95\% CI: $15.6-32.2 \%)$. The median duration of response (mDOR) was 5.0 months (0.9-19.1 months). Among the patients who responded to the treatment, 8 (32.0\%) patients had more than 6 months DOR. Subgroup analysis showed that the ORR of second-line treatment was 34\% (17/50), and that of third-line or more (the number of previous treatment lines was 3-8) was $13.8 \%$ (8/58). Similarly, 108 patients with cholangiocarcinoma (with FGFR2 gene fusion/ rearrangement) who had not previously received $F G F R$ inhibitors received pemigatinib $13.5 \mathrm{mg}$ (21-day cycle; 2 weeks on, 1 week off) in the FIGHT202 study (3). The ORR was $37 \%$, including 4 complete responses $(3.7 \%)$ and 36 partial responses (33.3\%). The mDOR was 8.08 months, and the median progression-free survival (mPFS) was 7.03 months (3). However, patients with FGFR mutations can not benefit from the treatment. By combining the above two studies, it can be seen that as to FGFR inhibitor in fusion/rearrangement patients, the ORR of the second-line therapy is at least $30 \%$, which is much higher than the $5 \%$ of the existing standard chemotherapy, and even not inferior to the ORR of the first-line standard regimen gemcitabine plus cisplatin (GC) (20-30\%). Therefore, it is expected that FGFR inhibitor is likely to be the first-line treatment and even perioperative treatment, although its application is post-line treatment now.

In addition, relative to $2020 \mathrm{v} 5$ version (4), the previous update of $2021 \mathrm{v} 2$ (5) version pointed out that pembrolizumab as the second-line treatment can be used to microsatellite instability-high (MSI-H)/mismatch repair deficient (dMMR) tumors/tumor mutational burden-high (TMB-H) tumors. For BRAF V600E mutant tumors, dabrafenib + trametinib can be used. It can be seen that the patient of biliary tract cancer is being finely divided through molecular phenotypic. In this update, microsatellite stability and TMB detection are proposed for unresectable total biliary tract tumors. It can be seen 
that cholangiocarcinoma has officially entered the era of precision treatment, and gene sequencing has gradually become a necessary examination. At present, the precise treatment targets recommended in the guideline include FGFR2, IDH1, BRAF, NTRK and MSI-H/dMMR/TMB-H. Interestingly, throughout the guideline, there is no nextgeneration sequencing (NGS) technology recommended, which is in line with the consistent and conservative recommendation style of NCCN. In contrast, ESMO's guidelines comprehensively recommended the application of NGS in cholangiocarcinoma this year.

As to the update of chemotherapy and immunotherapy, the progress of immunotherapy in cholangiocarcinoma is relatively slow. In addition to the MSI-H recommendations, the guidelines supplement that in some cases, nivolumab or lenvatinib + pembrolizumab can be used as class $2 \mathrm{~B}$ recommendations. The theoretical basis comes from two clinical trials. A phase 2 trial of nivolumab enrolled 46 patients with biliary tract cancer who had accepted at least the first-line therapy (6). The ORR was $11 \%$ (assessed by the independent expert committee). PFS and OS were 3.7 months and 14.2 months, respectively. According to the latest results of LEAP-005 (7), 31 patients were enrolled in the biliary tract cancer cohort. As of April 10, 2020, median time from first dose to data cutoff (DCO) was 9.5 months (range, 3.1-11.9 months), with 8 patients on treatment at DCO. Three (10\%) patients received PRs and18 (58\%) patients received SDs. ORR was $10 \%$ (95\% CI, 2-26\%), and DCR was $68 \%$ (95\% CI, 49-83\%). mDOR was 5.3 months (range, 2.1-6.2 months). mPFS was 6.1 months (95\% CI, 2.1-6.4 months). Median overall survival (mOS) was 8.6 months (95\% CI, 5.6-NR months). From the existing recommendations, the second-line ORR of immunotherapy may be better than the that of the standard chemotherapy FOLFOX. However, even combined with the antiangiogenic therapy, PD1 is not as effective as the result of hepatocellular carcinoma. It is still doubtful whether it can enter the first-line therapy. In addition, the treatment of chemotherapy combined with PD1 has no basis to be recommended.

Among all malignancies, liver cancer ranks sixth in incidence and fourth in mortality worldwide. Liver cancer caused 1,240,201 people's death worldwide in 2016, according to the Global Burden of Disease 2016. Since the approval of sorafenib for the first-line treatment of liver cancer, the treatment of liver cancer has opened a new era of targeted therapy. In the past decade, sorafenibbased regimens have been continuously explored for better survival benefits for patients. However, this year's NCCN guidelines include significant changes to the standard treatment of liver cancer. In the treatment strategy of liver cancer, antiangiogenic therapy combined with immunotherapy has become a new standard of first-line treatment. In the first-line systematic treatment scheme, there is a significant change that sorafenib and lenvatinib have been moved to "other recommended regimens", and the most preferred recommended scheme has been changed to atezolizumab + bevacizumab according to the phase 3 clinical data of IMBrave150. A total of 501 patients were enrolled in the IMbrave 150 study (8), 336 in the atezolizumab + bevacizumab group and 165 in the sorafenib group. The ORR of the atezolizumab + bevacizumab group and sorafenib group was $30 \%$ vs. $11 \%$, respectively. mDOR was 18.1 vs. 14.9 months, respectively. mOS was 19.2 months in the atezolizumab + bevacizumab group and 13.4 months in the sorafenib group. There is no doubt that this group has become a recognized standard firstline treatment in advanced HCC. The retreat of sorafenib to "other recommended regimens" is also an expected result. However, since the result of the study of LEAP002 (the control group is lenvatinib) has not been published, there is not enough data support that lenvatinib is also incorporated into "other recommended regimens" although it is understandable.

With regard to the application of atezolizumab + bevacizumab, the update points out that patients should conduct adequate endoscopic evaluation and management of esophageal varices within about 6 months before treatment. This update suggests the bleeding risk and applicable population of bevacizumab. In addition, in the update of first-line treatment, if antiangiogenic drugs cannot be used for various reasons, the single drug use of nivolumab can be considered, which comes from the results of randomized phase 3 study of CheckMate 459 (9). Nivolumab, whether ORR/PFS/OS, is not inferior to sorafenib and can be used as the first-line choice of single drug treatment. Especially in subgroup analysis, the survival of patients with PDL1 positive was significantly better than that of sorafenib group.

At present, many second-line clinical trials of immunotherapy are carrying out, but few can be recommended as clinical evidence. There are two main reasons. One is that immunotherapy is rapidly promoted to the first-line, but almost all the first-line regimens in the existing second-line studies are sorafenib/lenvatinib. Nivolumab and pembrolizumab can be recommended 
for patients who have not been treated with checkpoint inhibitors before. The evidence comes from similar trials which included patients after first-line sorafenib failure, such as Keynote 240 (10) or CheckMate 040 (11). There is little evidence of second-line treatment after the failure of the first-line immunotherapy. The second is the lack of successful clinical trials. For example, nivolumab + ipilimumab and pembrolizumab were adjusted from second-line optional regimens to other recommended regimens. The data of nivolumab + ipilimumab are from CheckMate 040, which is only a cohort study. In addition, the phase 3 clinical trial of pembrolizumab failed. Finally, due to the failure of Phase III clinical trial Keynote 240, pembrolizumab is only available for MSI-H hepatocellular carcinoma.

The above content is the main update of the medical treatment of hepatobiliary tumors this year, and everyone is looking forward to greater breakthroughs in treatment and more amazing research results being reported next year.

\section{Acknowledgments}

Funding: None.

\section{Footnote}

Provenance and Peer Review: This article was commissioned by the editorial office of Hepatobiliary Surgery and Nutrition. The article did not undergo external peer review.

Conflicts of Interest: Both authors have completed the ICMJE uniform disclosure form (available at https://hbsn. amegroups.com/article/view/10.21037/hbsn-21-355/coif). The authors have no conflicts of interest to declare.

Ethical Statement: The authors are accountable for all aspects of the work in ensuring that questions related to the accuracy or integrity of any part of the work are appropriately investigated and resolved.

Open Access Statement: This is an Open Access article distributed in accordance with the Creative Commons Attribution-NonCommercial-NoDerivs 4.0 International License (CC BY-NC-ND 4.0), which permits the noncommercial replication and distribution of the article with the strict proviso that no changes or edits are made and the original work is properly cited (including links to both the formal publication through the relevant DOI and the license). See: https://creativecommons.org/licenses/by-nc-nd/4.0/.

\section{References}

1. NCCN Clinical Practice Guidelines in OncologyHepatobiliary Cancers, Version 3. 2021. Available online: https://www.nccn.org/guidelines/guidelines-process/ transparency-process-and-recommendations

2. Javle MM, Roychowdhury S, Kelley RK, et al. Final results from a phase II study of infigratinib (BGJ398), an FGFR-selective tyrosine kinase inhibitor, in patients with previously treated advanced cholangiocarcinoma harboring an FGFR2 gene fusion or rearrangement. J Clin Oncol 2021;39:abstr 265 .

3. Abou-Alfa GK, Sahai V, Hollebecque A, et al. Pemigatinib for previously treated, locally advanced or metastatic cholangiocarcinoma: a multicentre, open-label, phase 2 study. Lancet Oncol 2020;21:671-84.

4. NCCN Clinical Practice Guidelines in OncologyHepatobiliary Cancers, Version 5. 2020. Available online: https://www.nccn.org/login?ReturnURL=https://www. nccn.org/professionals/physician_gls/pdf/hepatobiliary_ harmonized-africa.pdf

5. Benson AB, D'Angelica MI, Abbott DE, et al. Hepatobiliary Cancers, Version 2.2021, NCCN Clinical Practice Guidelines in Oncology. J Natl Compr Canc Netw 2021;19:541-65.

6. Kim RD, Chung V, Alese OB, et al. A phase 2 multiinstitutional study of nivolumab for patients with advanced refractory biliary tract cancer. JAMA Oncol 2020;6:888-94.

7. Lwin Z, Gomez-Roca C, Saada-Bouzid E, et al. LBA41 LEAP-005: Phase II study of lenvatinib (len) plus pembrolizumab (pembro) in patients (pts) with previously treated advanced solid tumours. Ann Oncol 2020;31:S1170.

8. Breder VV, Vogel A, Merle P, et al. IMbrave150: Exploratory efficacy and safety results of hepatocellular carcinoma (HCC) patients (pts) with main trunk and/ or contralateral portal vein invasion $(\mathrm{Vp} 4)$ treated with atezolizumab (atezo)+ bevacizumab (bev) versus sorafenib (sor) in a global Ph III study. J Clin Oncol 2021;39:abstr 4073.

9. Yau T, Park JW, Finn RS, et al. CheckMate 459: a randomized, multi-center phase III study of nivolumab (NIVO) vs sorafenib (SOR) as first-line (1L) treatment in patients (pts) with advanced hepatocellular carcinoma 
(aHCC). Ann Oncol 2019;30:v874-5.

10. Finn RS, Edeline J, Bouattour M, et al. Pembrolizumab (pembro) versus placebo (pbo) in patients (pts) with advanced hepatocellular carcinoma (aHCC) previously treated with sorafenib: Updated data from the randomized, phase 3 KEYNOTE-240 study. J Clin
Oncol 2021;39:abstr 4072.

11. Yau T, Kang YK, Kim TY, et al. Efficacy and safety of nivolumab plus ipilimumab in patients with advanced hepatocellular carcinoma previously treated with sorafenib: the CheckMate 040 randomized clinical trial. JAMA Oncol 2020;6:e204564.

Cite this article as: $\mathrm{Xu} \mathrm{L}$, Zhou J. Interpretation of NCCN guidelines for hepatobiliary tumors V3 update in 2021. HepatoBiliary Surg Nutr 2021;10(5):682-685. doi: 10.21037/ hbsn-21-355 